\title{
Thick branes in extra dimensions and suppressed dark couplings
}

\author{
Ricardo G. Landim and Thomas G. Rizzo \\ SLAC National Accelerator Laboratory, \\ 2575 Sand Hill Rd., Menlo Park, CA 94025, U.S.A. \\ E-mail: rlandim@slac.stanford.edu, rizzo@slac.stanford.edu
}

ABSTRACT: The nature of dark matter (DM) and how it may interact with the various fields of the Standard Model (SM) remains a mystery. In this paper we show that the interaction between new light dark matter mediators and the SM particles can be naturally suppressed if one employs a single, flat extra dimension (ED). In this setup, the SM fields are localized in a finite width 'fat' brane, similar to models of Universal Extra Dimensions (UED), while DM, in turn, is confined to a thin brane at the opposite end of the ED interval. Including brane localized kinetic terms on the fat brane for the mediator fields, the resulting coupling between the SM and these light mediators can be several orders of magnitude smaller than the corresponding ones between the mediators and DM which we assume to be a typical gauge coupling. We investigate the implications of this scenario for both vector (i.e, dark photon, DP) and scalar mediator fields in the 5-D bulk. In this setup kinetic mixing, which is usually employed to suppress light mediator couplings, is not required. Here we assume that the SM particles couple to the DP via their $B-L$ charges while the DP couples to the DM via a dark charge. Both the vector DP couplings and the corresponding Higgs portal couplings with the SM are shown to be natural small in magnitude with a size dependent on ratio of the 5-D compactification radius, $R^{-1} \sim 0.1-1 \mathrm{GeV}$, and the $\mathrm{SM}$ brane thickness, $L^{-1} \sim 2-10 \mathrm{TeV}$, a range chosen to avoid LHC and other experimental constraints. In this framework one can obtain the observed value of the DM relic abundance for a wide range of parameter choices, while the constrains due to direct DM detection and the invisible width of the Higgs do not impose significant challenges to the model. Finally, this mechanism can lead to distinct signatures in both present and upcoming experiments as it combines some common features of UED and DP models in a single ED setup.

Keywords: Phenomenology of Field Theories in Higher Dimensions

ArXiv EPrint: 1902.08339 


\section{Contents}

1 Introduction 1

2 Framework 2

3 Bulk gauge field mediator 3

3.1 Interactions 5

$\begin{array}{lll}3.2 & \text { Constraints on dark matter couplings } & 7\end{array}$

$\begin{array}{lll}\text { 3.2.1 Direct search constraints } & 7\end{array}$

3.2.2 Indirect constraints 9

4 Scalar mediator fields in the bulk $\quad \mathbf{1 2}$

4.1 The Higgs portal 13

$\begin{array}{lll}\text { 4.1.1 Higgs decay constraints } & 14\end{array}$

5 Discussion and conclusions $\quad 17$

\section{Introduction}

The nature of the dark matter (DM) remains a puzzling challenge to modern cosmology and particle physics. Although Weakly Interacting Massive Particles (WIMPs) are the most well-known candidates (see ref. [1] for a recent review), the lack of any positive signatures lead us to consider different scenarios, both from the theoretical and experimental pointof-view. Among the many possibilities, the interaction of DM with Standard Model (SM) particles through a new mediator field is a promising avenue of approach to the DM problem considering the diverse set of existing and planned direct detection, indirect detection and accelerator experiments [2]. A widely studied example of the approach is the dark photon model (DP) [3-10] (see ref. [11] for a review) wherein a new, relatively light, dark $\mathrm{U}(1)_{D}$ gauge field interacts with us via kinetic mixing with the SM hypercharge $\mathrm{U}(1)_{Y}$ field [12-17].

Many extensions of the SM appear by employing extra dimensions (ED). As is wellknown, the entire SM can be embedded in ED and this is most explicitly the case in the Universal Extra Dimension (UED) models, with either one [18] or two ED [19, 20], for example. In UED models the whole SM content is thus promoted to fields which propagate in the compact ED thus having Kaluza-Klein (KK) excitations. In 4-D, the lightest/zero mode of each KK tower of states is then identified with the correspondent SM particle, and the KK tower of excitations are heavier partner particles with the same spin as the zero mode. Searches for Supersymmetric (SUSY) [21, 22] particles at the LHC can then be re-interpretted to constrain the UED compactification radius, $L$, since both classes of models can have qualitatively similar phenomenology. Those searches imposed the current 
lower bound on the UED radius $L^{-1}>1.4-1.5 \mathrm{TeV}$ (for $\Lambda L \sim 5-35$, where $\Lambda$ UED is the cutoff scale) [23-25]. Other ED models have been employed to address multiple issues in particle physics such as the gauge hierarchy [26-31] and flavor problems [32-34]. In the context of ED, the DP model has been recently embedded in a flat, single ED, together with a DM candidate being either a scalar or a fermion $[35,36]$. In this scenario, the gauge boson in 4 -D acquires a mass due to the breaking of $\mathrm{U}(1)_{D}$ by boundary conditions (BC), thus no dark Higgs field is explicitly needed to generate the DP mass.

For the usual scalar singlet DM candidate, symmetric under $\mathbb{Z}_{2}$ [37-39], the interaction with the SM is only through the Higgs portal, whose implications have been examined in multiple contexts [40-60]. The size of the Higgs portal coupling is highly constrained by the bound on the invisible Higgs width for a general scalar, while for a DM candidate obtaining the observed relic density while also satisfying direct detection searches forces the parameter space to be even more highly constrained [61,62], requiring a highly tuned and very small Higgs portal coupling. The simplest version of this scenario is now almost, if not completely, excluded by these multiple constraints from several observations and experiments [63-82].

The small values of both the Higgs portal and DP couplings with the SM can appear naturally through an ED effect, as we shall see below. Here we will assume that the new $\mathrm{U}(1)_{D}$ gauge symmetry produces an interaction between SM and DM since the SM particles carry a $B-L$ charge while the DM has a dark charge. As we will see, for the DP in the bulk, there is no need for kinetic mixing to suppress this interaction. To be specific, we will introduce a single, flat ED, a DM candidate confined to one thin brane at one interval boundary while the SM is contained in a brane of finite thickness at the opposite end of the interval. The effect of this 'fat' brane is to suppress the couplings with the SM, leading to a natural small gauge or Higgs portal coupling.

This paper is organized as follows. Section 2 describes the basic framework that will be employed in this setup. In section 3 we consider a gauge field in the bulk and analyze the resulting couplings with the SM and DM. Also, we examine the constraints on the SM interactions with the DM particle from both direct and indirect observations. In section 4 we turn our attention to the case of a scalar mediator field in the bulk, derive the appropriate expressions to obtain the Higgs portal coupling and, in turn, analyze the invisible Higgs decay constraints in the ED context. Finally, section 5 is reserved for conclusions.

\section{Framework}

We will consider the case of a single flat ED, represented by an interval, with one thin brane localized at the $y=0$ boundary where the DM candidate is confined, and a 'fat' (i.e., thick) brane lying between $y=\pi r$ and $y=\pi R$, with a width $\pi(R-r) \equiv \pi L$, at the opposite boundary. The SM is contained within this fat brane and it is assumed that $L \ll R$. This scenario in which the $\mathrm{SM}$ experiences a TeV-scale ED is similar to models of UED [18], in which the SM content is promoted to fields which propagate through this compact ED of size $\pi L$, thus having KK excitation, whose eigenfunctions are $\sim \cos n y / L$ (or $\sim \sin n y / L)$, where $n$ labels the KK tower state. ${ }^{1}$

\footnotetext{
${ }^{1} \mathrm{SM}$ has also been assumed to be localized in branes in different contexts, as for instance in refs. [29, 83], while in refs. $[84,85]$ gravitons or fermion are localized in fat branes.
} 


\begin{tabular}{|c|cccc|}
\hline BM & I & II & III & IV \\
\hline$R^{-1}$ & $1 \mathrm{GeV}$ & $1 \mathrm{GeV}$ & $100 \mathrm{MeV}$ & $100 \mathrm{MeV}$ \\
$L^{-1}$ & $2 \mathrm{TeV}$ & $10 \mathrm{TeV}$ & $2 \mathrm{TeV}$ & $10 \mathrm{TeV}$ \\
\hline
\end{tabular}

Table 1. The different sets of parameters used in BM for the present study.

Throughout the paper, where needed to exemplify our calculations, we will consider four specific benchmark models (BM), whose assumed set of values for the compactification radius $R$ and the size of the UED $L$ are presented in table 1 . We note that these are merely representative and that many other choices of these parameters would yield results qualitatively similar to at least one of these cases.

\section{Bulk gauge field mediator}

We will first consider an abelian gauge field $V_{A}, A=0-3,5$ in the bulk, which interacts with both the DM and the SM. In the absence of kinetic mixing, the DP couples with DM and SM through the usual covariant derivative, however, it should not couple to the SM Higgs since such a coupling would influence, e.g., the $Z$ boson mass and other SM precision electroweak predictions which in turn are very well constrained [25]. One consistent way to introduce this coupling with SM particles is to use an anomaly-free symmetry under which baryons and/or leptons are charged, while the SM Higgs and gauge bosons are not. As is well-known, four symmetries may play this role without the presence of additional SM fermion fields (beyond RH-neutrinos) to cancel anomalies: the difference between baryon and lepton numbers $\left(U_{B-L}\right)$ and the three differences between the lepton numbers $\left(U_{L_{\mu}-L_{e}}\right.$, $U_{L_{e}-L_{\tau}}$ and $U_{L_{\mu}-L_{\tau}}$ ) [86-89]. DM, on the other hand, is assumed to not have any SM charges, in particular, a non-zero $B-L$ charge. $^{2}$ However, the DM does carry (a vectorlike) dark charge $Q_{D}$, therefore, we will assume that the DP couples to the combination $B-L+\lambda Q_{D}$ where $\lambda=1$ can be chosen without loss of generality. Thus the covariant derivative contains a term proportional to $\sim g_{5 D}\left(B-L+Q_{D}\right)$, where $g_{5 D}$ is 5 -D the dark gauge coupling. For the SM particles $B-L \neq 0$ and $Q_{D}=0$, while for DM, $B-L=0$ by assumption and we will assume that $Q_{D}=1$. No dark Higgs field is introduced as the symmetry is broken by the choice of $\mathrm{BC}$.

For completeness and to help with the numerics of the model construction we will also consider brane-localized kinetic terms (BLKT) at $y=0$, and within the thick SM brane [91]. The action for the DP field in the presence of these BLKT terms is then given by ${ }^{3}$

$$
S=\int d^{4} x \int_{0}^{\pi R} d y\left[-\frac{1}{4} V_{A B} V^{A B}-\frac{1}{4} V_{\mu \nu} V^{\mu \nu} \cdot \delta_{A} R \delta(y)-\frac{1}{4} V_{\mu \nu} V^{\mu \nu} \cdot \delta_{B} R \theta(y)\right]
$$

\footnotetext{
${ }^{2}$ Although it might have, as in the models of asymmetric dark matter [90].

${ }^{3}$ The induced kinetic term on the brane is effectively $4-\mathrm{D}$ at distances shorter than $R$, even in the case of a thick brane, as explained in [91].
} 
where $A$ is the 5 -D index and $\theta(y)$ is a step-function for the BLKT spread inside the thick brane [91], i.e.,

$$
\theta(y)=\alpha \quad \text { for } \pi r<y \leq \pi R, \quad \theta(y)=0 \quad \text { for } y<\pi r
$$

where $\alpha>0$. Although the parameter $\alpha$ is a new energy scale in the model, it is always multiplying the parameter $\delta_{B}$ and, as we shall see, variations of $\delta_{B} \alpha$ does not have a major effect on the results. In this sense, the energy scales associated with $R$ and $L$ are much more relevant than $\alpha$.

As usual, we expand the 5-D gauge field into a KK tower of states

$$
V^{\mu[5]}(x, y)=\sum_{n} v_{n}^{[5]}(y) V_{n}^{\mu[5]}(x),
$$

which yields the following equation of motion for $v_{n}(y)$ employing for convenience the $V^{5}=0$ gauge:

$$
\partial_{y}^{2} v_{n}+\left(m_{n}^{V}\right)^{2} v_{n}+\delta_{A} R \delta(y)\left(m_{n}^{V}\right)^{2} v_{n}+\delta_{B} R \theta(y)\left(m_{n}^{V}\right)^{2} v_{n}=0 .
$$

For $y<\pi r$, the step-fuction $\theta(y)=0$, leading to a simpler equation of motion [92], whose solution is found after applying the correspondent $\mathrm{BC}$ at $y=0$ employing the familiar solution for the equation of motion with a delta-function source (i.e., the continuity of the function and the discontinuity of its derivative) [35]

$$
v_{1, n}(y)=N_{n}^{V}\left[\cos \left(m_{n}^{V} y\right)-\frac{\delta_{A} x_{n}^{V}}{2} \sin \left(m_{n}^{V} y\right)\right] \quad 0 \leq y \leq \pi r
$$

where the resulting 4-D DP KK masses are given by $m_{n}^{V}=x_{n}^{V} / R$ and $N_{n}^{V}$ is a normalization factor. On the other hand, for $y>\pi r, \theta(y)=\alpha$ and we can define an effective mass parameter given by

$$
\bar{m}_{n}^{V}=m_{n}^{V} \sqrt{1+\delta_{B} \alpha R}
$$

such that the wave function in this region therefore must have the following form

$$
v_{2, n}(y)=A_{n} \cos \left(\bar{m}_{n}^{V} y\right)+B_{n} \sin \left(\bar{m}_{n}^{V} y\right) \text {. }
$$

The coefficients $A_{n}$ and $B_{n}$ are then found through the standard requirement that the wavefunction and its derivative should be continuous at the $y=\pi r$ 'boundary'. After applying this condition we obtain (here $\mathrm{a}^{\prime}$ denotes a derivative with respect to the $y$ co-ordinate)

$$
v_{2, n}(y)=v_{1, n}(\pi r) \cos \left[\bar{m}_{n}^{V}(y-\pi r)\right]+\frac{v_{1, n}^{\prime}(\pi r)}{\bar{m}_{n}^{V}} \sin \left[\bar{m}_{n}^{V}(y-\pi r)\right] \quad \pi r \leq y \leq \pi R .
$$

The presence of the BLKT inside the thick brane is responsible for the existence of this distinct wave function in this region. If $\delta_{B} \alpha=0$ the effective masses $\bar{m}_{n}^{V}$ would reduce 

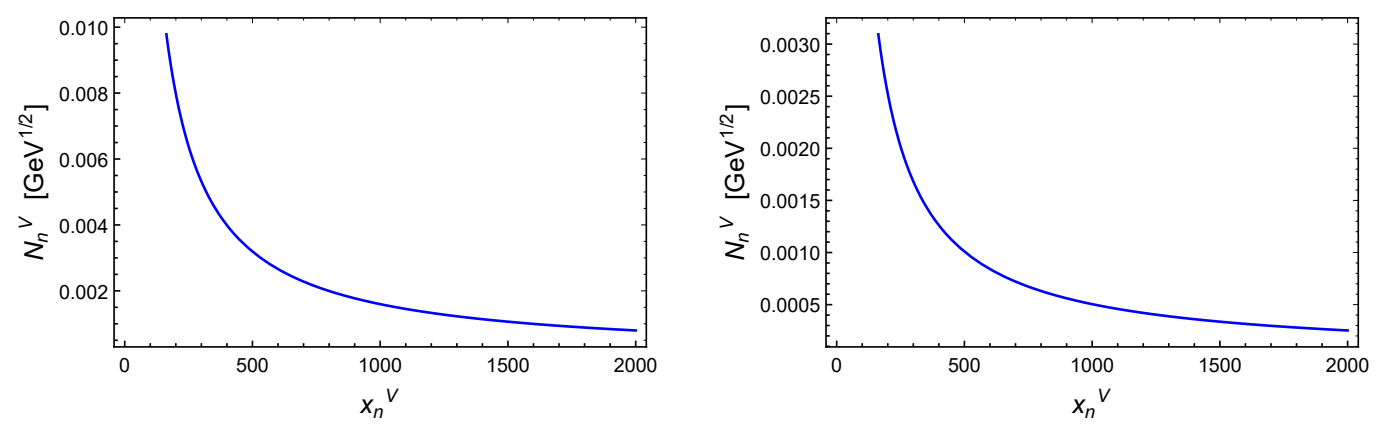

Figure 1. Normalization $N_{n}^{V}$ as a function of $x_{n}^{V}$, for BM II (left) and IV (right) with $\delta_{A}=\delta_{B}=1$ and $\alpha=1 \mathrm{GeV}$. BM I (III) presents basically the same behavior as BM II (IV) which are not shown.

to the same expression as that for the physical masses $m_{n}^{V}$ and the solution as presented in eq. (3.8) would be $v_{1, n}(y)$. Finally, using the BLKT modified orthogonality relations

$$
\begin{aligned}
\int_{0}^{\pi R} d y\left[1+\delta_{A} R \delta(y)+\delta_{B} R \theta(y)\right] v_{m}(y) v_{n}(y) & =\delta_{m, n} \\
\int_{0}^{\pi R} d y \partial_{y} v_{m}(y) \partial_{y} v_{n}(y) & =m_{m}^{V} m_{n}^{V} \delta_{m, n},
\end{aligned}
$$

we can obtain the overall normalization factors for the wave functions:

$$
\begin{aligned}
\frac{2}{R}\left(N_{n}^{V}\right)^{-2}= & {\left[\frac{1}{x_{n}^{V}}-\left(\frac{\delta_{A} x_{n}^{V}}{2}\right)^{2}\right] \sin \left(\frac{2 \pi r x_{n}^{V}}{R}\right)+\delta_{A} \cos \left(\frac{2 \pi r x_{n}^{V}}{R}\right)+\frac{2 \pi r}{R}\left[\left(\frac{\delta_{A} x_{n}^{V}}{2}\right)^{2}+1\right] } \\
& +\delta_{A}-\left[\frac{2 \pi L}{R}+\frac{\sin \left(\bar{m}_{n}^{V} \pi L\right)}{\bar{m}_{n}^{V} R}\right] \csc ^{2}\left(\bar{m}_{n}^{V} \pi L\right)\left(v_{1, n}(\pi r)\right)^{2}\left(1+\delta_{B} \alpha R\right) .
\end{aligned}
$$

The behavior of eq. (3.10) is depicted in figure 1, for some representative BM values of $R$ and $L$ as given in table 1 . The $\mathrm{BC} v_{2, n}(\pi R)=0$ leads to the root equation which determines the physical DP KK tower masses:

$$
\tan \left(\bar{m}_{n}^{V} \pi L\right)=-\bar{m}_{n}^{V} \frac{v_{1, n}(\pi r)}{v_{1, n}^{\prime}(\pi r)},
$$

whose solutions determine the physically allowed (quantized) values of $x_{n}^{V}$. All previous results for a thin brane [35,36] are recovered in the limit that $\delta_{B} \rightarrow 0$ and $r \rightarrow R$. Again we note that the lightest DP KK particle is massive due to our choice of the BC, without the need of a dark Higgs vacuum expectation value (VEV).

\subsection{Interactions}

The couplings between the tower of DP KK states and DM is now simply given by the value of the DP KK tower field wavefunctions evaluated at the DM brane, i.e., the coupling constants $g_{D, n}=g_{D} N_{n}^{V} / N_{1}^{V}$, where $g_{D} \equiv g_{5 D} N_{1}^{V}$ is defined to be the 4-D gauge dark coupling and $N_{1}^{V}$ is the normalization of the lowest DP KK state $(n=1)$. On the other 
hand, the interaction between the DP and a (zero-mode) SM field, $\psi$, localized 'inside' the thick brane is given by the integral

$$
\int_{\pi r}^{\pi R} d y V^{\mu} J_{\mu}
$$

where $J_{\mu}$ is the SM current in this version of UED. Since we are interested in the interaction with the conventional SM particles, the zero mode of a generic SM field within the thick brane, $\psi$, is simply $\psi / \sqrt{\pi L}$, where $(\pi L)^{-1 / 2}$ is the familiar normalization of the UED fields. The 4-D gauge couplings of the KK gauge fields due to the effect of the thick brane is then given by the integral

$$
\begin{aligned}
g_{D, n}^{E D} & \equiv g_{5 D} \int_{\pi r}^{\pi R} d y \frac{v_{2, n}(y)}{\pi L} \\
& =\frac{g_{D} v_{1, n}(\pi r)}{N_{1}^{V} \bar{m}_{n}^{V} \pi L} \tan \left(\frac{\bar{m}_{n}^{V} \pi L}{2}\right),
\end{aligned}
$$

where to obtain the last line we employed the root equation (3.11) above. For the lower states of the KK tower, since $L \ll R$, we can expand the tangent in the above expression and use the root equation (3.11) in this small mass limit. The result is

$$
g_{D, n}^{E D} \simeq g_{D} \pi \frac{L}{R} x_{n}^{V} \frac{N_{n}^{V}}{2 N_{1}^{V}}\left[\sin \left(m_{n}^{V} \pi r\right)+\frac{\delta_{A}}{2} x_{n}^{V} \cos \left(m_{N}^{V} \pi r\right)\right] .
$$

For small values of $x_{n}^{V}$ the effect of the extra dimension is thus to reduce the dark coupling by a factor of $\sim L / R$. With a compactification scale of size $R^{-1} \sim 0.1-1 \mathrm{GeV}$ and the size of the UED as $L^{-1} \sim 2-10 \mathrm{TeV}$, for instance, the interaction with SM particles is thus a factor of $\sim 10^{-(3-4)}$ smaller than is the coupling with DM. For more massive particles in the KK tower, the coupling with SM fields is suppressed since $N_{n}^{V} \sim\left(x_{n}^{V}\right)^{-1}$ and thus from eq. (3.13) we see that a large $m_{n}^{V}$ leads directly to a decrease in the coupling. The full behavior of eq. (3.13) is shown in figure 2, where we used the root equation to determine the KK masses. BM I and II (III and IV) produce the same general pattern and whose difference can be seen directly from eq. (3.14), although it holds for the full expression. For purposes of demonstration only we have assumed that $g_{D}=1$ here and in the subsequent analysis below. We note that BM II and IV have $L / R$ values ten times smaller than the correspondent ones with $L^{-1}=2 \mathrm{TeV}$ (i.e., BM I and III), thus, in order to compensate for this reduction, ten times as many KK states (via the roots $x_{n}^{V}$ ) are required to match the same behavior. Although leading to non-renormalizable interactions in 4-D, it is interesting that the previous result (i.e., the $L / R$ suppression of couplings) generalizes to the case of higher powers of the 5-D mediator field. Consider a generic 5-D field $\Phi$, then, having interactions with the SM fields that scale as $\Phi^{\beta}$; this leads to interactions of the form $\phi^{\beta} \int d y\left(v_{2, n}(y)\right)^{\beta} \sim \phi^{\beta}\left(\frac{\pi L x_{n}^{V}}{R}\right)^{\beta}$, in the limit of small KK masses for diagonal couplings, where $\phi$ is the lowest KK 4 -D field.

In figure 3 we depict the general behavior of the SM couplings of the gauge KK states for different values of $\delta_{A}$ and $\delta_{B}$ in BM I and III; these results are for illustrative purposes 

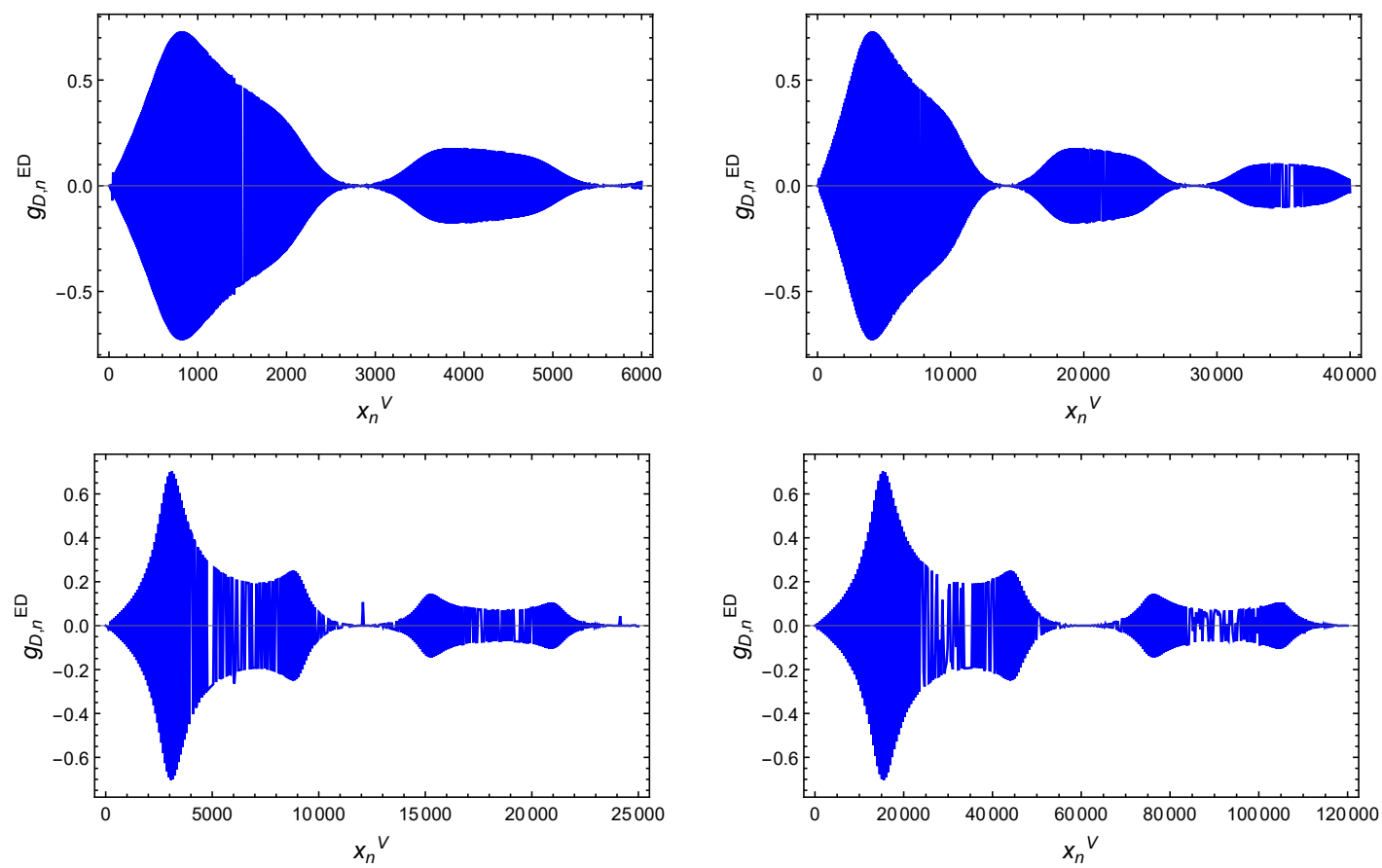

Figure 2. Oscillatory behavior of the gauge KK couplings as a function of $x_{n}^{V}$, for $\delta_{A}=\delta_{B}=1$, $\alpha=1 \mathrm{GeV}$ and $g_{D}=1$ for purposes of demonstration, for BM I (top left), BM II (top left), BM III (bottom left) and BM IV (bottom right).

only. Increasing $\delta_{A}$, the couplings are also increased as can be seen from eq. (3.14). On the other hand, larger values of $\delta_{B} \alpha$ lead to an increase in the effective masses $\bar{m}_{n}^{V}$ thus making the whole oscillatory pattern begin earlier, i.e., the first big peak for BM III is near $x_{n}^{V} \sim 1500$ for $\delta_{B} \alpha=1 \mathrm{GeV}$ while near $x_{n}^{V} \sim 500$ for $\delta_{B} \alpha=10 \mathrm{GeV}$. Figure 4 presents the KK couplings for BM I with a small value of $\delta_{B} \alpha$ which is seen to have the opposite behavior, that is, it reduces the effective masses $\bar{m}_{n}^{V}$ thus leading to a smoother oscillatory pattern which reach maxima at higher values of the roots (compare the BM I in figure 4 with the ones in figures 2 and 3). As $\delta_{B} \alpha$ increases the oscillatory pattern becomes sharper with oscillations beginning at smaller values of the $x_{n}^{V}$. This pattern can be seen in figure 5 , where we considered a large value of $\delta_{B} \alpha$.

\subsection{Constraints on dark matter couplings}

We now explicitly consider the interactions of the brane localized DM candidate through the DP KK tower to the SM; the DM here is assumed to be a complex scalar field for simplicity. The couplings of the DP KK tower with the DM is just $g_{D, n} \equiv g_{D} N_{n}^{V} / N_{1}^{V}$, while for the SM it is given by $g_{D, n}^{E D}$ as described in eq. (3.13).

\subsubsection{Direct search constraints}

The mass and couplings of the DM particle are constrained by both direct and indirect experimental searches. In order for the DM not to annihilate into a pair of DP particles (which occurs in an s-wave and thus is excluded by Planck results on the CMB [93] for 

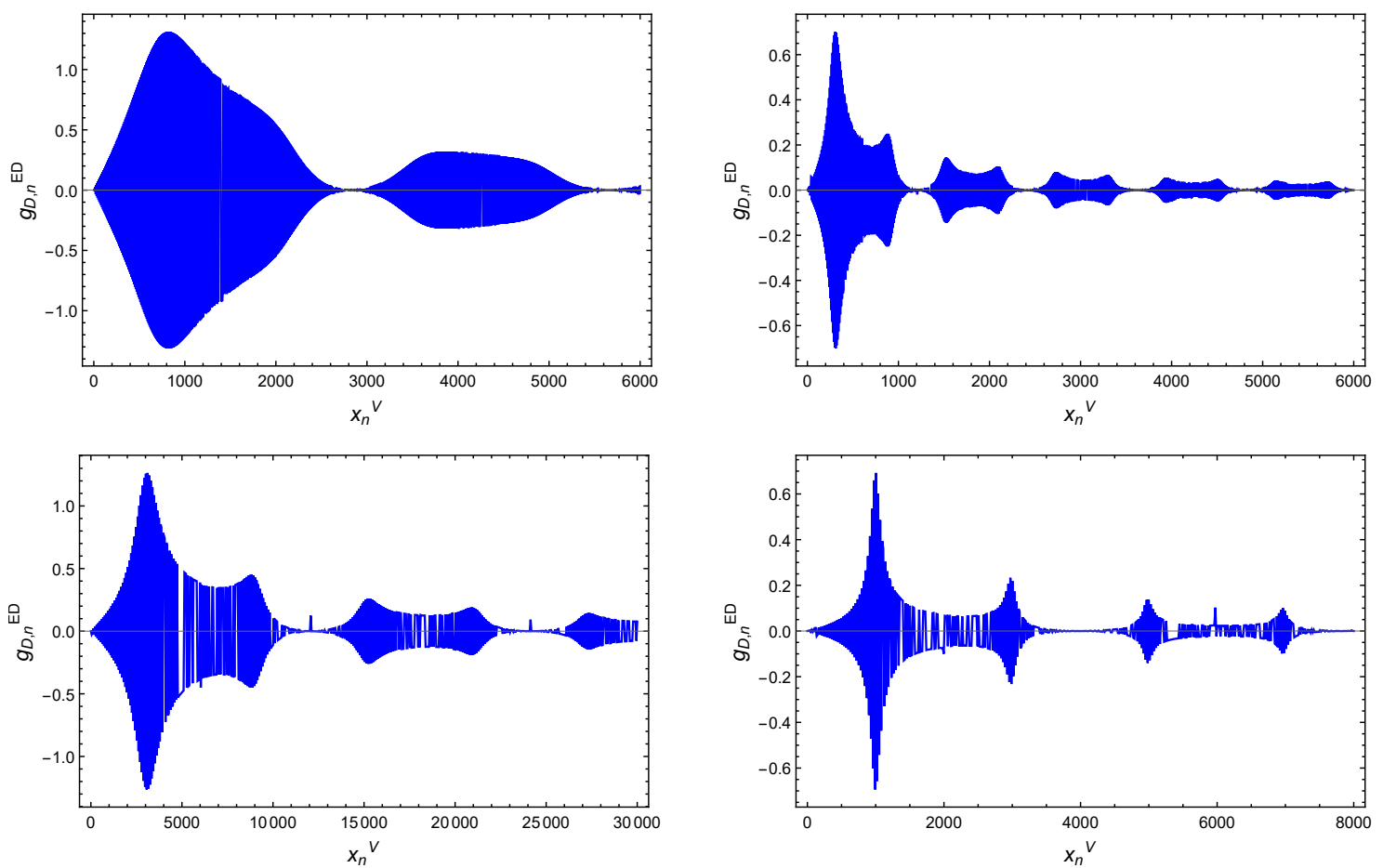

Figure 3. Gauge couplings as a function of $x_{n}^{V}$, for $\delta_{A}=10$ (left) or $\delta_{B} \alpha=10 \mathrm{GeV}$ (right), while the other parameters are the same as figure 2, for BM I (top) and BM III (bottom).
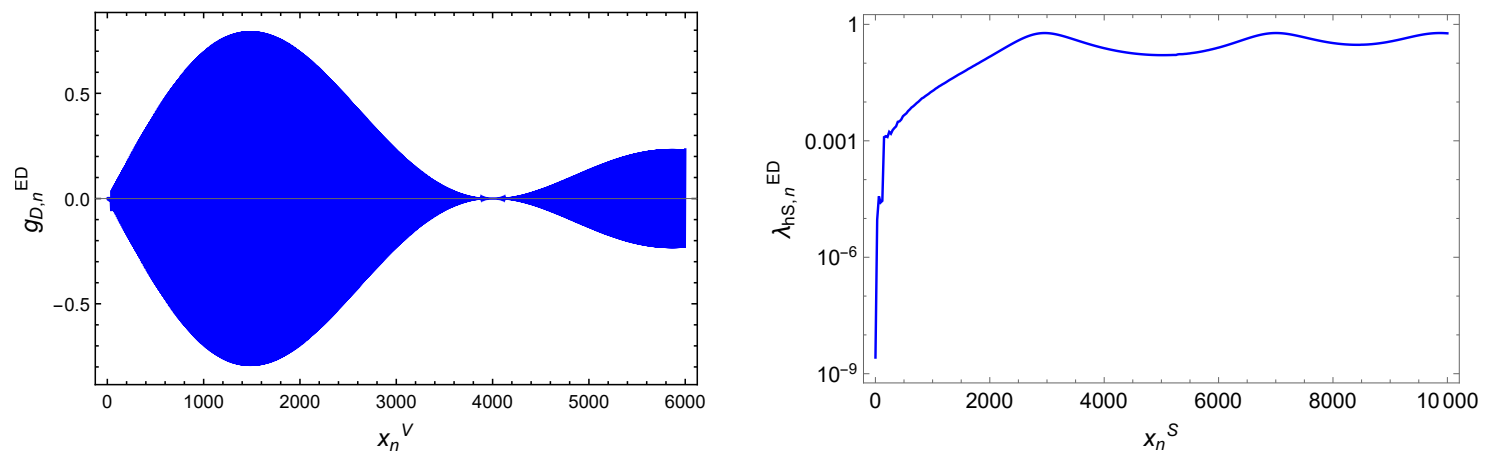

Figure 4. Left (right): gauge (Higgs) couplings as a function of $x_{n}^{V}$, for $\delta_{B} \alpha=10^{-2} \mathrm{MeV}$, while the other parameters are the same as figure 2, for BM I.

DM masses in our range of interest), it should be lighter than the lightest DP KK state. Thus, the DM mass must be smaller than $x_{1}^{V} / R$, where $x_{1}^{V}$ is the lowest KK root, which generally lies in the range $\sim 0.2-0.5$ for the BMs considered here. The mass scale for the $\mathrm{DM}$ is, therefore, set by both the compactification radius $R$ and the value of the first root obtained from the DP KK tower mass eigenvalue equation above. Due to the magnitude of the DM mass ( $\sim 20-400 \mathrm{MeV}$, as we will see below) and the corresponding small recoil energies in direct detection, DM scattering off electrons provides greater sensitivity [2]; the 

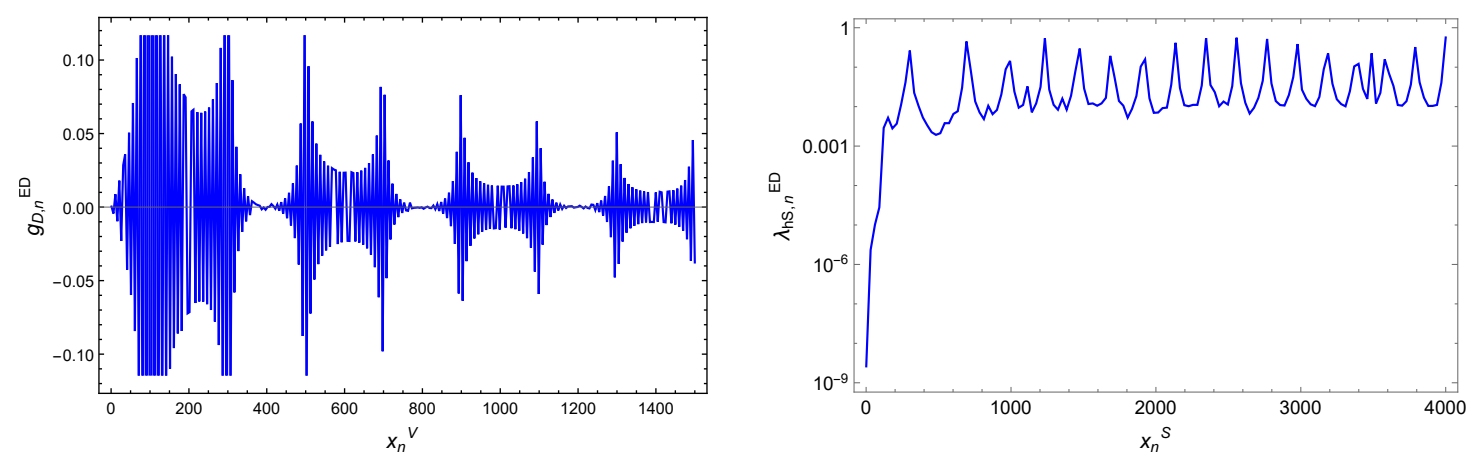

Figure 5. Left (right): gauge (Higgs) couplings as a function of $x_{n}^{V}$, for $\delta_{B} \alpha=10 \mathrm{TeV}$, while the other parameters are the same as figure 2, for BM I.
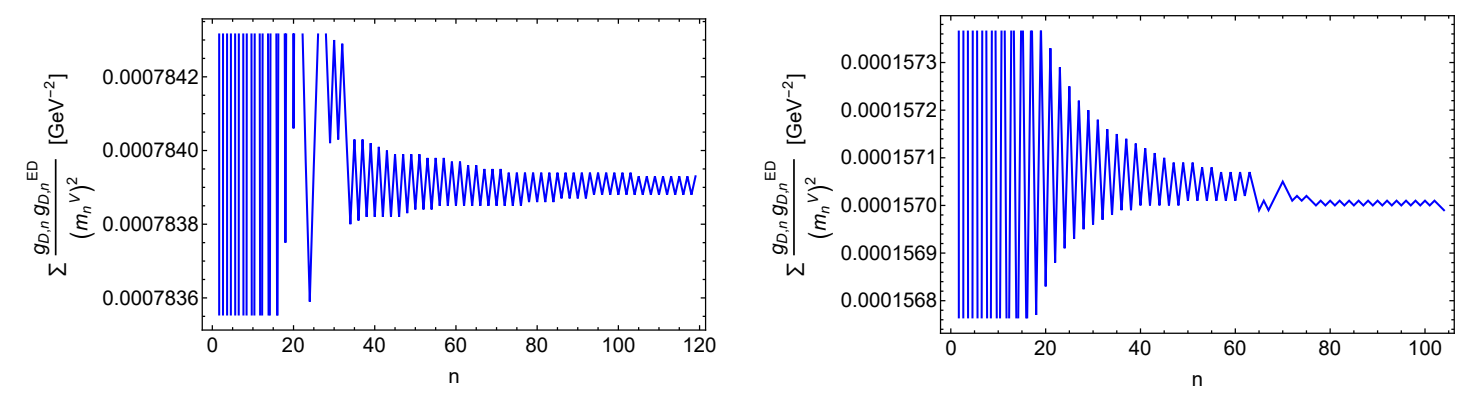

Figure 6. Sum of the first $n$ terms in eq. (3.15) (the behavior is qualitatively the same for the sum in eq. (3.16)), for BM I (left) and BM II (right). For the other BM points the pattern is similar. It can be seen that these sums converges quickly, being relatively constant after $n \sim 50$. Small jumps are due to round off errors in the calculation.

scattering cross section in this case is [94-96]

$$
\sigma_{e}=\frac{\mu^{2}}{4 \pi}\left(\sum_{n} \frac{g_{D, n} g_{D, n}^{E D}}{\left(m_{n}^{V}\right)^{2}}\right)^{2},
$$

where a form factor of unity has been assumed and the reduced mass $\mu=m_{e} m_{D M} /\left(m_{e}+\right.$ $\left.m_{D M}\right) \sim m_{e}$, since $m_{D M}^{2} \gg m_{e}^{2}$. Given the couplings and masses from the above considerations the KK sum that appears in this expression converges quite rapidly with only the first few terms being numerically significant (see figure 6). The resulting scattering cross section has been/can be constrained using the results from XENON10 [97], XENON100 [98], DarkSide-50 [99] or SENSEI [100]. The corresponding values of the elastic scattering cross sections for the four different BM points are shown in table 2.

\subsubsection{Indirect constraints}

Considering the standard scenario of thermal freeze-out, given the DM mass range of interest, the resulting final states from DM pair annihilation can be $e^{+} e^{-}$and $\mu^{+} \mu^{-}$, as well as three generations of (essentially) massless neutrinos since the DP couples to $B-L$. Note that as mesons do not have either baryon nor lepton number, a pair of pions is not an accessible channel of annihilation. The cross section away from a KK resonance can 
be approximately expanded in powers of the relative DM annihilation, $v^{2}$, and is given by $\sigma v \approx a+b v^{2}$, where, for a vector mediator and a complex scalar DM, the annihilation cross section is dominantly a p-wave with coefficients $a=0$ and for a particular final state fermion $f[95]$

$$
b_{f}=\frac{m_{D M}^{2}}{6 \pi} \sqrt{1-\frac{m_{f}^{2}}{m_{D M}^{2}}}\left(1-\frac{m_{f}^{2}}{2 m_{D M}^{2}}\right)\left(\sum_{n} \frac{g_{D, n} g_{D, n}^{E D}}{\left(m_{n}^{V}\right)^{2}-4 m_{D M}^{2}}\right)^{2},
$$

where $m_{f}$ is the mass of the final states. The sum above converges rapidly (see figure 6) because the couplings $g_{D, n}^{E D}$ go to zero rather quickly for higher roots $x_{n}^{V}$ in addition to the presence of the DP tower propagator masses which rapidly decreases the possible contribution of heavier DP states.

We employ the following expression to determine the value of the coupling $g_{D}$ which gives the observed value of relic density of DM [95]

$$
\Omega h^{2} \simeq \frac{x_{f} 1.07 \times 10^{9} \mathrm{GeV}^{-1}}{g_{*}^{1 / 2} m_{P l}\left(a+3 b / x_{f}\right)},
$$

where $m_{P l}$ is the Planck mass and $x_{f} \equiv m_{D M} / T_{f}$ is the usual ratio between the DM mass and the temperature at the freeze-out, which is taken to be $x_{f}=20$. The effective number of degrees of freedom for the range of DM masses of interest here $(20-400 \mathrm{MeV}$, as we shall see) is $g_{*} \simeq 10.75$, since the temperature at the freeze-out is $\sim 1-20 \mathrm{MeV}$. Setting $\Omega h^{2}=0.12$ [93] our results for $g_{D}$ are found in table 2. The lightest DP KK mass is shown here together with the value for the DM mass. A slightly heavier DM particle can be considered, provided that $m_{D M}<m_{1}^{V}$ remains valid, although the results are practically unchanged. Smaller values of $m_{D M}$ increase the value of $g_{D}$ needed to obtain the observed DM relic abundance, as seen in eq. (3.16). Recall that $g_{D}$ is defined here to be the coupling of the lowest DP KK tower state to the DM and that all of the values of $\alpha_{D}=g_{D}^{2} / 4 \pi$ shown here are $<0.4$.

The sum appearing in eq. (3.16) is qualitatively the same for BM I and III (or BM II and IV). This comes from the fact that the differences in the coupling $g_{D, n}^{E D}$ (roughly one order of magnitude, and whose behavior are very well described by eq. (3.14)) is compensated by presence of the different DP masses in the denominator of eq. (3.16). Although the DM masses are one order of magnitude smaller for BM III (IV) than for BM I (II), leading apparently to smaller cross sections, this reduction is compensated for by the normalization of the first root, i.e., $N_{1}^{V}$, which is roughly three times larger for BM I (II) than in BM III (IV) (see figure 1). The most 'promising' among these BM appears to be BM I and III, since even with smaller DM masses, which in turn leads to larger couplings. Increasing or decreasing the thick brane parameter $\delta_{B} \alpha$ (e.g., $\delta_{B} \alpha=1 / 2 \mathrm{GeV}$ or $\delta_{B} \alpha=10 \mathrm{GeV}$ ) does not significantly change these numerical results. A large increase of the combination $\delta_{B} \alpha$ $\left(\delta_{B} \alpha=10 \mathrm{TeV}\right)$ has also no significant impact on the results presented, being the most relevant change for BM I, in which the scattering cross section is decreased by almost one order of magnitude. Variations in the parameter $\delta_{A}$, on the other hand, do lead to some numerical modifications here. If $\delta_{A}=1 / 2$ is assumed, the numerics are unchanged but 


\begin{tabular}{|c|c|c|c|c|}
\hline $\mathrm{BM}$ & I & II & III & IV \\
\hline \multicolumn{5}{|l|}{$\delta_{A}=1$} \\
\hline$m_{1}^{V}[\mathrm{MeV}]$ & 430 & 430 & 43 & 43 \\
\hline$m_{D M}[\mathrm{MeV}]$ & 400 & 400 & 40 & 40 \\
\hline$g_{D}$ & 0.89 & 1.98 & 0.96 & 2.15 \\
\hline$\sigma_{e}\left[\mathrm{~cm}^{2}\right]$ & $1.1 \times 10^{-40}$ & $1.1 \times 10^{-40}$ & $1.6 \times 10^{-38}$ & $1.6 \times 10^{-38}$ \\
\hline \multicolumn{5}{|l|}{$\delta_{A}=1 / 2$} \\
\hline$m_{1}^{V}[\mathrm{MeV}]$ & 460 & 460 & 46 & 46 \\
\hline$m_{D M}[\mathrm{MeV}]$ & 430 & 430 & 43 & 43 \\
\hline$g_{D}$ & 0.88 & 2.00 & 0.95 & 2.12 \\
\hline$\sigma_{e}\left[\mathrm{~cm}^{2}\right]$ & $8.8 \times 10^{-41}$ & $9.6 \times 10^{-41}$ & $1.2 \times 10^{-38}$ & $1.2 \times 10^{-38}$ \\
\hline \multicolumn{5}{|l|}{$\delta_{A}=10$} \\
\hline$m_{1}^{V}[\mathrm{MeV}]$ & 230 & 230 & 23 & 23 \\
\hline$m_{D M}[\mathrm{MeV}]$ & 200 & 200 & 20 & 20 \\
\hline$g_{D}$ & 0.88 & 1.95 & 0.93 & 2.08 \\
\hline$\sigma_{e}\left[\mathrm{~cm}^{2}\right]$ & $1.1 \times 10^{-39}$ & $1.1 \times 10^{-39}$ & $1.4 \times 10^{-37}$ & $1.4 \times 10^{-37}$ \\
\hline \multicolumn{5}{|l|}{$\delta_{B} \alpha=1 / 2 \mathrm{GeV}$} \\
\hline$m_{1}^{V}[\mathrm{MeV}]$ & 430 & 430 & 43 & 43 \\
\hline$m_{D M}[\mathrm{MeV}]$ & 400 & 400 & 40 & 40 \\
\hline$g_{D}$ & 0.81 & 1.98 & 0.96 & 2.14 \\
\hline$\sigma_{e}\left[\mathrm{~cm}^{2}\right]$ & $7.7 \times 10^{-41}$ & $1.1 \times 10^{-40}$ & $1.5 \times 10^{-38}$ & $1.5 \times 10^{-38}$ \\
\hline \multicolumn{5}{|l|}{$\delta_{B} \alpha=10 \mathrm{GeV}$} \\
\hline$m_{1}^{V}[\mathrm{MeV}]$ & 430 & 430 & 43 & 43 \\
\hline$m_{D M}[\mathrm{MeV}]$ & 400 & 400 & 40 & 40 \\
\hline$g_{D}$ & 0.81 & 1.98 & 0.96 & 2.14 \\
\hline$\sigma_{e}\left[\mathrm{~cm}^{2}\right]$ & $7.8 \times 10^{-41}$ & $1.1 \times 10^{-40}$ & $1.5 \times 10^{-38}$ & $1.5 \times 10^{-38}$ \\
\hline \multicolumn{5}{|l|}{$\delta_{B} \alpha=10 \mathrm{TeV}$} \\
\hline$m_{1}^{V}[\mathrm{MeV}]$ & 430 & 430 & 43 & 43 \\
\hline$m_{D M}[\mathrm{MeV}]$ & 400 & 400 & 40 & 40 \\
\hline$g_{D}$ & 0.72 & 1.98 & 0.94 & 2.14 \\
\hline$\sigma_{e}\left[\mathrm{~cm}^{2}\right]$ & $3.0 \times 10^{-41}$ & $1.1 \times 10^{-40}$ & $1.3 \times 10^{-38}$ & $1.5 \times 10^{-38}$ \\
\hline
\end{tabular}

Table 2. 4-D dark coupling $g_{D}$ needed to obtain the observed DM relic density, with $\delta_{A}=1 / 2,1$ or 10 (with $\delta_{B} \alpha=1 \mathrm{GeV}$ ), and $\delta_{B} \alpha=1 / 2,10 \mathrm{GeV}$ or $10 \mathrm{TeV}$ (with $\delta_{A}=1$ ). The maximum value for the DM mass and the lightest DP mass are also shown. For BM II and IV the DM particle is lighter than muons, thus the only channels accessible is $e^{+} e^{-}$and neutrinos. The cross section for the scattering off of an electron by a DM particle is shown in the last row, using the same gauge coupling needed to satisfy the DM relic abundance. 
if instead one chooses $\delta_{A}=10$, the results are slightly modified; we gather all of these variations in table 2 . Note that the values of the couplings $g_{D}$ are slightly reduced when $\delta_{A}=10$, however, the scattering cross section $\sigma_{e}$ in this case is found to be roughly one order of magnitude smaller, thus BM III and IV are essentially excluded employing the current direct search experimental results [97-100].

\section{Scalar mediator fields in the bulk}

We now consider instead a scalar field $S$ in the bulk and investigate whether the ED can suppress the corresponding coupling with SM fields as we saw above in the case of a bulk gauge field. A real scalar field without an additional $\mathbb{Z}_{2}$ symmetry would give rise to potentially dangerous mass mixing terms between the scalar and the SM Higgs, thus spoiling the agreement of the Higgs couplings measurements with SM expectations. Therefore the imposition of a $\mathbb{Z}_{2}$ symmetry would be required. However, a real scalar with an exact $Z_{2}$ symmetry would be stable and could be a possible DM candidate. Since this particle is not assumed to be DM here, it must decay into, e.g., our DM candidate. These issues can be avoided if the scalar field is complex with a Higgs-like potential, couples to the DP and obtain a VEV. This VEV, in turn, gives a mass to the DP in the bulk, but this induces only order one changes in the results obtained in the previous sections.

However, for simplicity we will consider the following mechanism for a real scalar field in the bulk with a $\mathbb{Z}_{2}$ symmetry, without loss of generality. The action for the scalar field in the bulk is (see footnote in the gauge field case)

$$
\begin{aligned}
& S=\int d^{4} x \int_{0}^{\pi R} d y {\left[\frac{1}{2} \partial_{A} S \partial^{A} S+\frac{1}{2} \partial_{\mu} S \partial^{\mu} S \cdot \delta_{S} R \delta(y)+\frac{1}{2} \partial_{\mu} S \partial^{\mu} S \cdot \delta_{T} R \theta(y)\right] } \\
&+\int d^{4} x \int_{\pi r}^{\pi R} d y \lambda_{5 h S} S^{2}|H|^{2}
\end{aligned}
$$

where $H$ is the SM Higgs field and as before we have added BLKTs and the step-function $\theta(y)$ as given by eq. (3.2). In principle the scalar field may have a bulk mass as well as quartic potential terms, however, for simplicity, we will ignore this possibility as they will not play any essential role in what follows.

We then expand the 5-D scalar field in a KK tower of states and the equation of motion is found to be similar to the one for the gauge field above. However, now we have a bulk mass term but only for the region within the thick brane, i.e., $\pi r<y \leq \pi R$, given by $m_{b}^{2} \equiv \lambda_{5 h S} v_{h}^{2} /(\pi L)$, arising from the SM Higgs VEV. Note the presence of the square of the factor $(\pi L)^{-1 / 2}$ being the normalization of the zeroth mode of SM Higgs field as in UED and here $v_{h}$ is the Higgs VEV.

The physical masses are determined expanding the 5-D scalar field in the action (4.1) in a KK tower of states. The masses will have contributions due to the fields in the two distinct regions in the ED, i.e., in the region $R>y>r$ and $r>y>0$. The physical KK 
masses that appears in the 4-D action are thus given by

$$
\begin{aligned}
\left(m_{p, n}^{S}\right)^{2} \delta_{m, n} \equiv & \int_{0}^{\pi r} d y \partial_{y} s_{1, m}(y) \partial_{y} s_{1, n}(y)+\int_{\pi r}^{\pi R} d y \partial_{y} s_{2, m}(y) \partial_{y} s_{2, n}(y) \\
& +m_{b}^{2} \int_{\pi r}^{\pi R} d y s_{2, m}(y) s_{2, n}(y) \\
= & \left(m_{n}^{S}\right)^{2}+\lambda_{h S, m, n}^{E D} v_{h}^{2},
\end{aligned}
$$

where the first integrals yield the quantity $\left(m_{n}^{S}\right)^{2}$ after integrating by parts and we defined the 4-D couplings $\lambda_{h S, m, n}^{E D}$ as

$$
\lambda_{h S, m, n}^{E D} \equiv \lambda_{5 h S} \int_{\pi r}^{\pi R} d y \frac{s_{2, m}(y) s_{2, n}(y)}{\pi L} .
$$

The equation of motion for $s_{1}(y)$ is found to be

$$
\partial_{y}^{2} s_{1, n}+\left(m_{n}^{S}\right)^{2} s_{1, n}+\left(m_{n}^{S}\right)^{2} \delta_{S} R \delta(y) s_{1, n}=0,
$$

whose solution is the same as eq. (3.5), with the trivial replacement $\delta_{A} \rightarrow \delta_{S}$. For $\pi r<y \leq \pi R$, however, the equation of motion for $s_{2}(y)$ is now

$$
\partial_{y}^{2} s_{2, n}+\left(m_{n}^{S}\right)^{2} s_{2, n}+m_{b}^{2} s_{2, n}+\left(m_{n}^{S}\right)^{2} \delta_{T} \alpha R s_{2, n}=0
$$

where we may define an effective mass as

$$
\left(\bar{m}_{n}^{S}\right)^{2}=\frac{\left(x_{n}^{S}\right)^{2}}{R^{2}}\left(1+\delta_{T} \alpha R\right)+m_{b}^{2} .
$$

Using the above definition, the solution for $s_{2, n}$, the normalization $N_{n}^{S}$ and the root equation have the same structure of eqs. (3.8), (3.10) and (3.11), respectively, with the replacements $\delta_{A} \rightarrow \delta_{S}, \delta_{B} \rightarrow \delta_{T}, \bar{m}_{n}^{V} \rightarrow \bar{m}_{n}^{S}$ and $x_{n}^{V} \rightarrow x_{n}^{S}$. Of course, the solutions of the root equation are quantitatively different from the gauge field case. Once the quantized masses $m_{n}^{S}$ and the 4-D $\lambda_{h S, m, n}^{E D}$ are found through the root equation, the physical masses are straightforwardly determined.

\subsection{The Higgs portal}

The coupling between the SM Higgs and scalar mediator field $S$ is given by the Higgs portal interaction, $\lambda_{5 h S}|H|^{2} S^{2}$. We split the effect of the extra dimension on the coupling into two different contributions: that arising from the particles at the same KK level ' $n$ ' $\left(m_{m}^{S}=m_{n}^{S}\right)$ and for that contributions arising between different levels of the KK tower $\left(m_{m}^{S} \neq m_{n}^{S}\right)$. These are seen to arise from the integral over the ED in eq. (4.3). The integral for $m=n$, after applying the root equation, is given by

$$
\lambda_{h S, n}^{E D}=\frac{\lambda_{h S} s_{1, n}^{2}}{2\left(N_{1}^{S}\right)^{2}}\left[\csc ^{2}\left(\bar{m}_{n}^{S} \pi L\right)-\frac{1}{\bar{m}_{n}^{S} \pi L} \cot \left(\bar{m}_{n}^{S} \pi L\right)\right]
$$



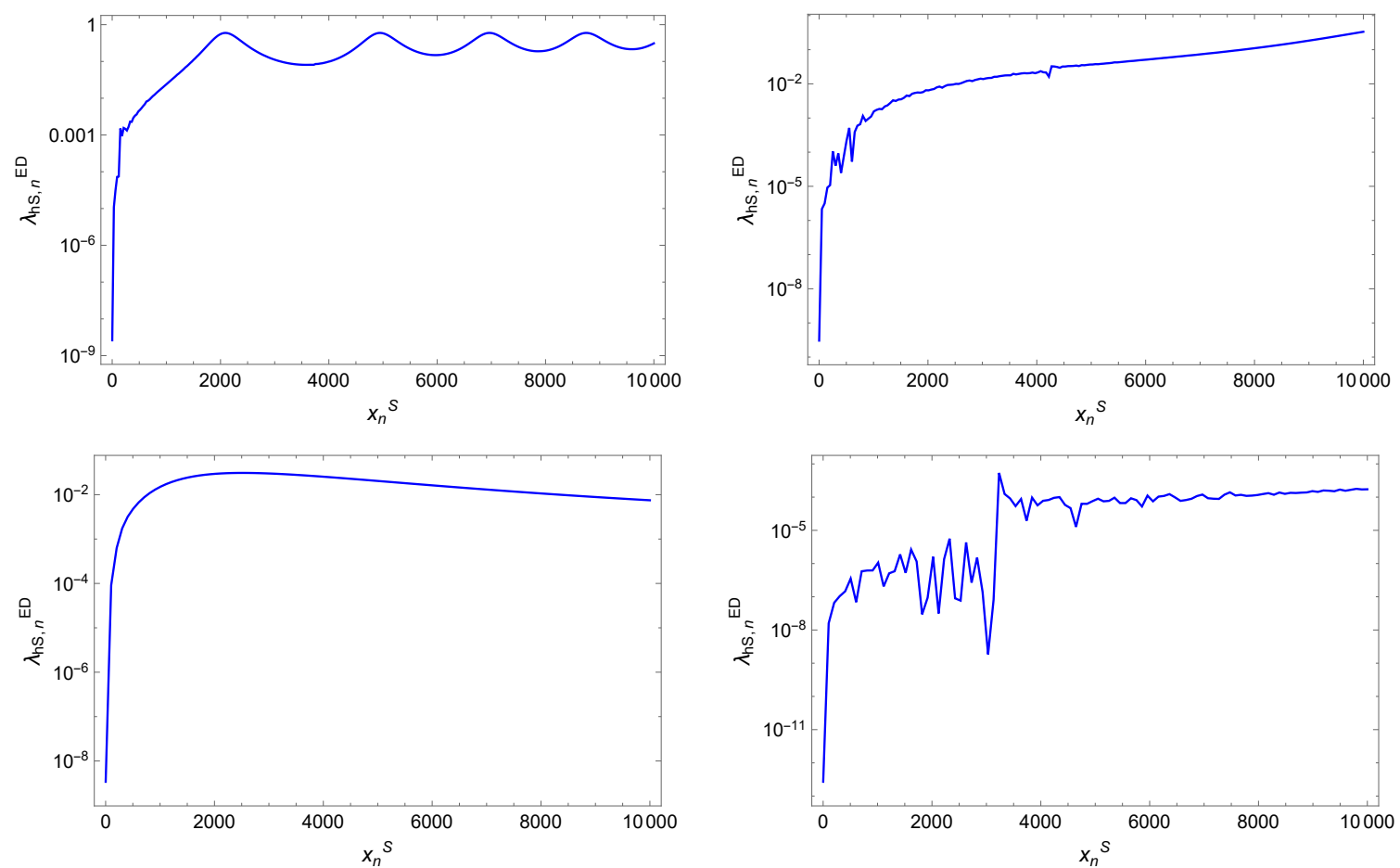

Figure 7. Higgs portal couplings as a function of $x_{n}^{S}$, for $\delta_{S}=\delta_{T}=1, \alpha=1 \mathrm{GeV}$ and $\lambda_{h S}=1$, for BM I (top left), BM II (top left), BM III (bottom left) and BM IV (bottom right) as described above.

while the integral for $m \neq n$ gives

$$
\lambda_{h S, m, n}^{E D}=\frac{\lambda_{h S} s_{1, m} s_{1, n}}{\left(N_{1}^{S}\right)^{2} \pi L}\left[\frac{\bar{m}_{n}^{S} \cot \left(\bar{m}_{n}^{S} \pi L\right)-\bar{m}_{m}^{S} \cot \left(\bar{m}_{m}^{S} \pi L\right)}{\left(\bar{m}_{m}^{S}\right)^{2}-\left(\bar{m}_{n}^{S}\right)^{2}}\right],
$$

and where $s_{1, m(n)}$ are here understood to be evaluated at $y=\pi r$. As before, we may define a 4-D quartic coupling as $\lambda_{h S} \equiv \lambda_{5 h S}\left(N_{1}^{S}\right)^{2}$. Since the bulk scalar mass is not small, the effective mass $\bar{m}_{m}^{S}$ is not as well, therefore we cannot expand the trigonometric functions in the above expressions, as we have done in the gauge field case.

The numerical results obtained from eq. (4.7) are depicted in figure 7, where the pattern of BM I and II (or III and IV) are explained by the same reasoning as described for the gauge interaction in the last section. Different values of $\delta_{S}$ and $\delta_{T} \alpha$ are shown in figures 4 and 8 . The behavior of the off-diagonal couplings can be seen in figure 9 . The couplings here are seen to oscillates around zero, but are generally increasing in absolute value for heavier masses.

\subsubsection{Higgs decay constraints}

The lower members of the bulk scalar KK tower are generally lighter than the Higgs boson itself, so that the Higgs can decay into a pair of potentially neutral long-lived particles of the KK tower. The most recent constraint for the Higgs decay into invisible particles is given by the ATLAS experiment [101]. The $h \rightarrow S_{n} S_{m}$ decay rate can be calculated 

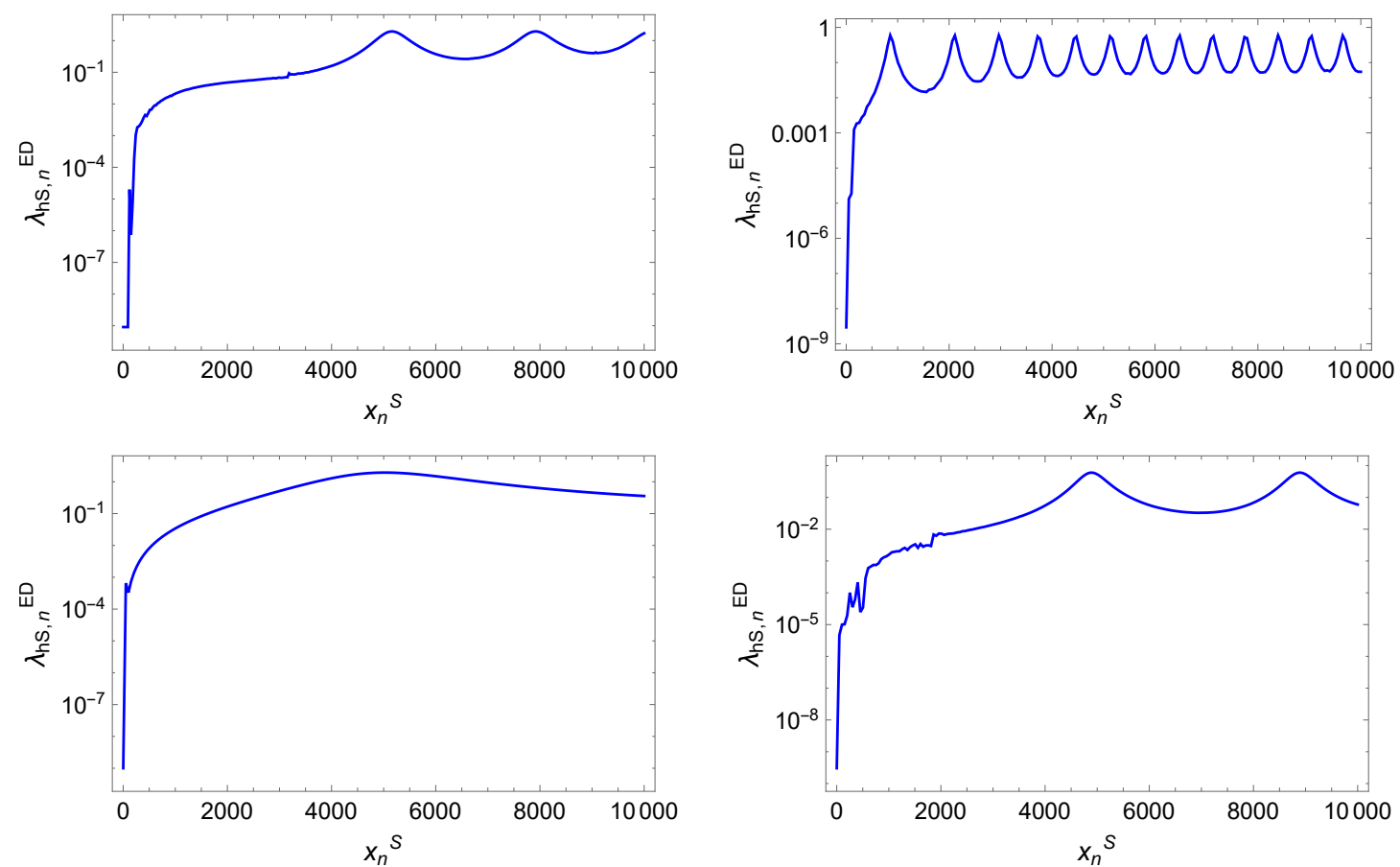

Figure 8. Higgs portal couplings as a function of $x_{n}^{S}$, for $\delta_{S}=10$ (left) or $\delta_{T} \alpha=10 \mathrm{GeV}$ (right), but with the other parameters unchanged, for BM I (top) and BM II (bottom).
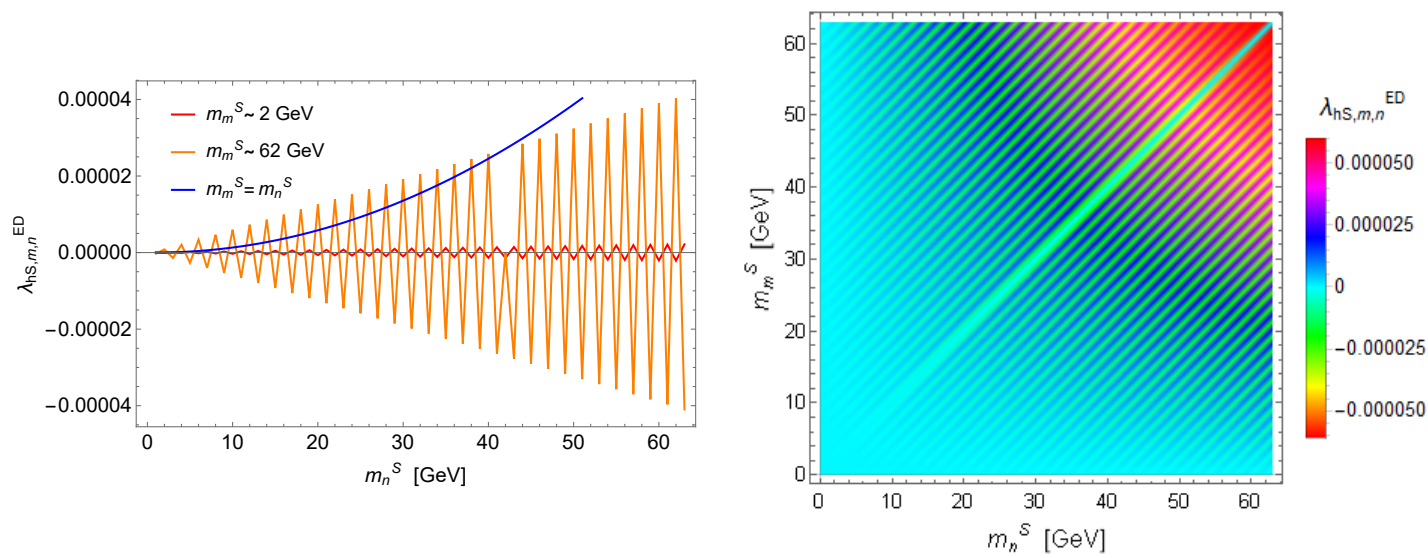

Figure 9. Left: the coupling $\lambda_{h S, m, n}^{E D}$, for BM I, with $m$ fixed for different values of $m_{m}^{S}$ and $\lambda_{h S}=1$. Right: coupling $\lambda_{h S, m, n}^{E D}$ up to $m_{m(n)}^{S}=62 \mathrm{GeV}$.

employing the standard result [25]

$$
d \Gamma_{m, n}=\frac{1}{32 \pi^{2}}|\mathcal{M}|^{2} \frac{\left|\mathbf{p}_{m, n}\right|}{m_{h}^{2}} d \Omega,
$$

where $m_{h}=125 \mathrm{GeV}$ is the SM Higgs mass, the outgoing momentum is

$$
\left|\mathbf{p}_{m, n}\right|=\left|\mathbf{p}_{n, m}\right|=\frac{\left[\left(m_{h}^{2}-\left(m_{m}+m_{n}\right)^{2}\right)\left(m_{h}^{2}-\left(m_{m}-m_{n}\right)^{2}\right)\right]^{1 / 2}}{2 m_{h}^{2}},
$$




\begin{tabular}{|c|cccc|}
\hline BM & I & II & III & IV \\
\hline $\mathcal{B}\left(h \rightarrow S_{m}+S_{n}\right)$ & $3.6 \times 10^{-3}$ & $3.7 \times 10^{-5}$ & 0.87 & $3.5 \times 10^{-7}$ \\
\hline
\end{tabular}

Table 3. Branching fractions for the invisible Higgs decay into two KK scalars summed over all kinematically allowed states with $\delta_{S}=\delta_{T}=1, \alpha=1 \mathrm{GeV}$ and $\lambda_{h S}=1$.

and the integral over the solid angle is $4 \pi$ for $m \neq n$ and $2 \pi$ for $m=n$. The invariant amplitude $|\mathcal{M}|$ is $2 \lambda_{n}^{E D} v_{h}$ for $m=n$ and $\lambda_{m, n}^{E D} v_{h}$ for $m \neq n$, where $v_{h}$ is the Higgs VEV. Here we take $\lambda_{h S}=1$ for simplicity. Then, the total decay rate due to the tower of kinematically accessible scalar KK particles is given by $\Gamma^{E D}=\sum_{m, n} \Gamma_{m, n}$. When $m=n$ the Higgs can decay into particle pairs with individual masses up to $\sim 62.5 \mathrm{GeV}$ while when $m \neq n$, the masses of the outgoing KK particles can range up to $\simeq m_{h}$. The branching fractions for this invisible decay $\mathcal{B}\left(h \rightarrow S_{m}+S_{n}\right)=\Gamma^{E D} /\left(\Gamma_{H}^{S M}+\Gamma^{E D}\right)$, where $\Gamma_{H}^{S M} \simeq 4 \mathrm{MeV}$ is the SM Higgs total width [25], summed over kinematically accessible KK states are shown in table 3 for the four BM models described above in table 1 .

The differences between the branching fractions for the various BM arise from two distinct factors: $i$ ) the difference in the quartic coupling, as seen, e.g., in the BMs with $L^{-1}=10 \mathrm{TeV}$ (BM II and IV), which are two or three orders of magnitude smaller than the ones where $L^{-1}=2 \mathrm{TeV}$; and $i i$ ) the total number of KK states that need to be summed over which can contribute to Higgs decay. If $R^{-1}=1 \mathrm{GeV}$ (BM I and II) there are $\sim 62$ states with masses below $62.5 \mathrm{GeV}$, while for $R^{-1}=100 \mathrm{MeV}$ (BM III and IV) there are $\sim 10$ times as many. This fact is seen in the final numerics (BM III has a larger decay rate than $\mathrm{BM} \mathrm{I}$ ) once there are more $\mathrm{KK}$ tower states that contribute to the sum. The expected larger decay rate obtained for BM IV in comparison to BM II (given $i i$ above) is compensated by the smaller couplings that appear in this case (by roughly three orders of magnitude), thus leading to an overall smaller branching fraction instead of a potentially expected larger one. Clearly BM III is disfavored by these results.

In order to elucidate the role of the BLKT in the previous results, we consider BM II as an example and analyze the normalized decay rate for different values of both $\delta_{S}$ and $\delta_{T} \alpha$. For $\delta_{S}=10, \mathcal{B}\left(h \rightarrow S_{m}+S_{n}\right)=1.4 \times 10^{-2}$, summing over all kinematically accessible KK states, while for $\delta_{S}=1 / 2$ we obtain instead $\mathcal{B}\left(h \rightarrow S_{m}+S_{n}\right)=7.0 \times 10^{-5}$. Taking $\delta_{T} \alpha=10 \mathrm{GeV}$, e.g., the corresponding result is $\mathcal{B}\left(h \rightarrow S_{m}+S_{n}\right)=3.4 \times 10^{-7}$, while for $\delta_{T} \alpha=10 \mathrm{TeV}$ the branching ratio is $\mathcal{B}\left(h \rightarrow S_{m}+S_{n}\right)=2.8 \times 10^{-9}$. These differences in the branching fractions arise mainly due to the order of magnitude of the effective Higgs portal couplings, which are an order smaller for $\delta_{S}=1$ than for $\delta_{S}=10$ (see the left bottom panel of figure 8 for $m=n$, though the two result are similar for $m \neq n$ ). For larger $\delta_{T} \alpha$ the couplings are similar when $m=n$ (compare the right top panel of figure 7 with the right bottom panel of figure 8), but are also one order of magnitude smaller when $m \neq n$, as can be seen in figure 10, thus leading to a smaller branching fraction, since the couplings for $m=n$ are in general smaller than the ones for $m \neq n$. From all these previous results, one sees that all benchmark models but BM III are far below the LHC constraints on the invisible Higgs decay $\mathcal{B}(h \rightarrow i n v) \lesssim 0.2[61,102]$. In this scenario, because the scalar field couples 

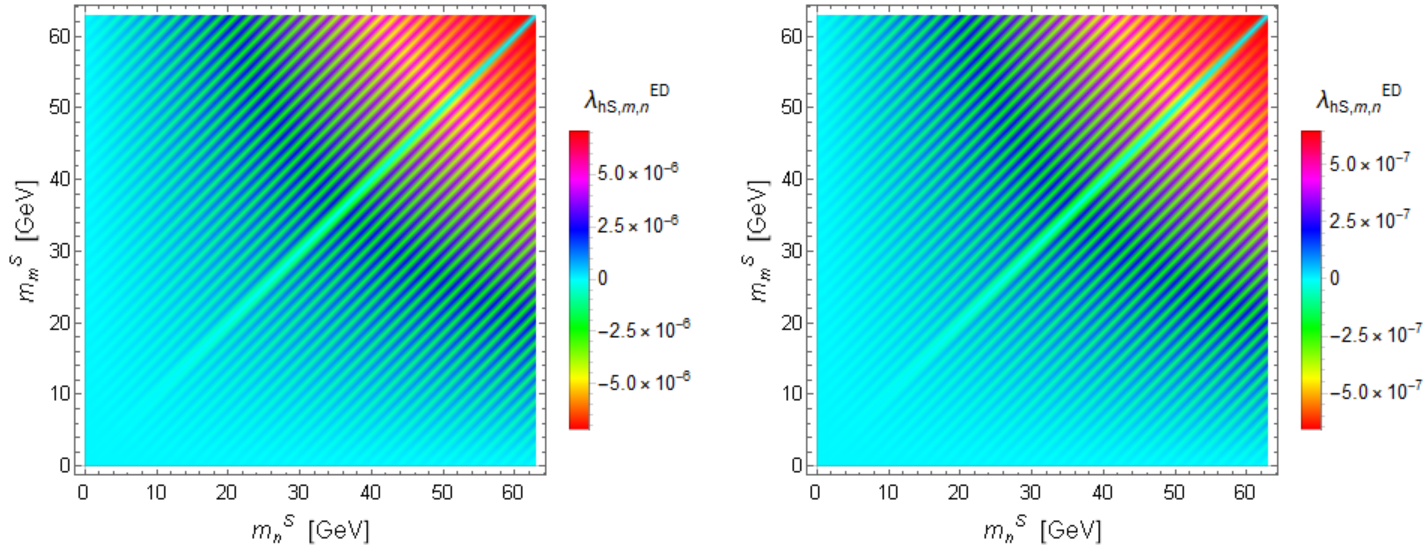

Figure 10. Couplings $\lambda_{h S, m, n}^{E D}$ up to $m_{m(n)}^{S}=62 \mathrm{GeV}$ for BM II, with $\delta_{T} \alpha=1 \mathrm{GeV}$ (left) and $\delta_{T} \alpha=10 \mathrm{GeV}$ (right). The other parameters are the same as in figure 7 .

to DM through a Higgs portal-like coupling, the annihilation process proceeds only through loops and so its contribution is negligible in the determination of the DM relic abundance.

\section{$5 \quad$ Discussion and conclusions}

In this paper we have presented a mechanism that naturally suppresses the various dark couplings with the SM which occur through a bulk mediator by employing a flat, single ED. The SM is confined in a thick brane while the DM is localized on the other (thin) brane at the opposite end of the interval. For both vector and scalar mediator fields in the bulk the mechanism is qualitatively similar: the coupling with the SM is significantly reduced in comparison to the corresponding coupling with DM, which we explicitly examined for both the gauge and the Higgs portal interactions. The sizes of the couplings in turn depend upon the ratio of the 5 -D compactification radius, $R^{-1} \sim 0.1-1 \mathrm{GeV}$, and the SM brane thickness, $L^{-1} \sim 2-10 \mathrm{TeV}$, whose range was chosen to avoid LHC and other experimental constraints. In this scenario, a kinetic mixing between the DP and the SM hypercharge gauge field is not needed once the DM carries a dark charge and the SM particles carry a $B-L$ charge. This special feature arises from a BLKT within the thick brane since the wave function in that small region is different from the one in the rest of the ED.

The observed DM relic abundance was obtained in the four BM considered but whose annihilation channels and rates clearly rely on the DM mass. Since the DM mass is assumed to be smaller than that of the lightest DP KK state, its value is bounded by the compactification radius $R$ and the smallest DP mass/root $x_{1}^{V}$.

The entire approach we considered here relies upon the use of BLKTs in order to obtain different mass parameters $\left(m_{n}^{V(S)}\right.$ and $\left.\bar{m}_{n}^{V(S)}\right)$ in the two regions of ED (the thick brane region, and the rest of the ED). However, if only a scalar mediator field was present in the bulk, the interaction with the Higgs would still induce an effective mass (4.6) different from $m_{n}^{S}$, leading to distinct wave functions in the two regions of the ED. Therefore, in this hypothetical situation, the resulting difference in the wave functions in these two regions 
could arise solely due to the Higgs VEV, therefore not necessarily requiring the BLKT. Even so, as presented before, a gauge field in the bulk does require a BLKT in order to suppress the couplings with SM.

Direct detection experiments employing DM scattering off of electrons do not currently exclude much of the parameter space of this model but it will be probed in further detail in the near future. The masses of the DP KK states in 4-D are essentially generated by our choice of BC, without the need of a dark Higgs field. When a scalar mediator field is present in the bulk the limits on invisible SM Higgs decays impose important constrains on the corresponding scalar couplings to the SM but only one of our BM is found to not satisfy this bound. We found that the thick brane mechanism considered here naturally reduces the Higgs portal couplings as well as the DP gauge couplings without the need of $\mathrm{BC}$ to force the vanishing of the Higgs portal in the vicinity of the SM fields (as described in refs. $[35,36])$ and the requirement that the kinetic mixing is described by a small parameter.

Although it was worth to study the behavior of the Higgs portal coupling due to the addition of an extra scalar field in the bulk, it does not influence the constraints obtained for our DM candidate, thus the presence of this scalar is not mandatory in order for the model to be self-consistent.

Potential signatures of the present model include a combination of searches for UEDlike particles and KK towers of the DP states. Missing energy searches at LHC, from the cascade decay of excited KK modes of the SM particles, constrain the compactification radius $L^{-1}$ as in the UED models. In addition to these searches, for heavier DP particles of the KK tower, which are at least twice as heavy as DM, the resulting cascade decay also gives a missing energy signature. For the lightest DP (which is slightly heavier than DM) the main decay final states are likely to be charged leptons or missing energy. Therefore, the present approach can lead to distinct signatures in ongoing and upcoming experiments, since it combines some common features of UED and DP models in an ED.

\section{Acknowledgments}

RGL is supported by CNPq under the grant 208206/2017-5. The work of TGR was supported by the Department of Energy, Contract DE-AC02-76SF00515.

Open Access. This article is distributed under the terms of the Creative Commons Attribution License (CC-BY 4.0), which permits any use, distribution and reproduction in any medium, provided the original author(s) and source are credited.

\section{References}

[1] G. Arcadi et al., The waning of the WIMP? A review of models, searches and constraints, Eur. Phys. J. C 78 (2018) 203 [arXiv:1703.07364] [INSPIRE].

[2] M. Battaglieri et al., US Cosmic Visions: New Ideas in Dark Matter 2017: Community Report, in U.S. Cosmic Visions: New Ideas in Dark Matter, College Park, MD, U.S.A., March 23-25, 2017 (2017) [arXiv: 1707.04591] [INSPIRE]. 
[3] D. Feldman, B. Körs and P. Nath, Extra-weakly Interacting Dark Matter, Phys. Rev. D 75 (2007) 023503 [hep-ph/0610133] [INSPIRE].

[4] D. Feldman, Z. Liu and P. Nath, The Stueckelberg Z-prime Extension with Kinetic Mixing and Milli-Charged Dark Matter From the Hidden Sector, Phys. Rev. D 75 (2007) 115001 [hep-ph/0702123] [INSPIRE].

[5] M. Pospelov, A. Ritz and M.B. Voloshin, Secluded WIMP Dark Matter, Phys. Lett. B 662 (2008) 53 [arXiv:0711.4866] [inSPIRE].

[6] M. Pospelov, Secluded U(1) below the weak scale, Phys. Rev. D 80 (2009) 095002 [arXiv:0811.1030] [INSPIRE].

[7] H. Davoudiasl, H.-S. Lee and W.J. Marciano, Muon Anomaly and Dark Parity Violation, Phys. Rev. Lett. 109 (2012) 031802 [arXiv:1205.2709] [InSPIRE].

[8] H. Davoudiasl, H.-S. Lee and W.J. Marciano, 'Dark' Z implications for Parity Violation, Rare Meson Decays and Higgs Physics, Phys. Rev. D 85 (2012) 115019 [arXiv:1203.2947] [INSPIRE].

[9] R. Essig et al., Working Group Report: New Light Weakly Coupled Particles, in Proceedings, 2013 Community Summer Study on the Future of U.S. Particle Physics:

Snowmass on the Mississippi (CSS2013), Minneapolis, MN, U.S.A., July 29-August 6, 2013 (2013) [arXiv: 1311.0029] [INSPIRE].

[10] E. Izaguirre, G. Krnjaic, P. Schuster and N. Toro, Analyzing the Discovery Potential for Light Dark Matter, Phys. Rev. Lett. 115 (2015) 251301 [arXiv:1505.00011] [INSPIRE].

[11] D. Curtin, R. Essig, S. Gori and J. Shelton, Illuminating Dark Photons with High-Energy Colliders, JHEP 02 (2015) 157 [arXiv:1412.0018] [INSPIRE].

[12] B. Holdom, Two U(1)'s and Epsilon Charge Shifts, Phys. Lett. 166B (1986) 196 [INSPIRE].

[13] B. Holdom, Searching for $\epsilon$ Charges and a New U(1), Phys. Lett. B 178 (1986) 65 [INSPIRE].

[14] K.R. Dienes, C.F. Kolda and J. March-Russell, Kinetic mixing and the supersymmetric gauge hierarchy, Nucl. Phys. B 492 (1997) 104 [hep-ph/9610479] [INSPIRE].

[15] F. Del Aguila, The Physics of z-prime bosons, Acta Phys. Polon. B 25 (1994) 1317 [hep-ph/9404323] [INSPIRE].

[16] K.S. Babu, C.F. Kolda and J. March-Russell, Leptophobic U(1)'s and the $R_{b}-R_{c}$ crisis, Phys. Rev. D 54 (1996) 4635 [hep-ph/9603212] [INSPIRE].

[17] T.G. Rizzo, Gauge kinetic mixing and leptophobic $Z^{\prime}$ in $E_{6}$ and $\mathrm{SO}(10)$, Phys. Rev. D 59 (1998) 015020 [hep-ph/9806397] [INSPIRE].

[18] T. Appelquist, H.-C. Cheng and B.A. Dobrescu, Bounds on universal extra dimensions, Phys. Rev. D 64 (2001) 035002 [hep-ph/0012100] [InSPIRE].

[19] G. Burdman, B.A. Dobrescu and E. Ponton, Six-dimensional gauge theory on the chiral square, JHEP 02 (2006) 033 [hep-ph/0506334] [INSPIRE].

[20] E. Ponton and L. Wang, Radiative effects on the chiral square, JHEP 11 (2006) 018 [hep-ph/0512304] [INSPIRE].

[21] ATLAS collaboration, Search for squarks and gluinos in events with isolated leptons, jets and missing transverse momentum at $\sqrt{s}=8$ TeV with the ATLAS detector, JHEP 04 (2015) 116 [arXiv:1501.03555] [INSPIRE]. 
[22] ATLAS collaboration, Search for squarks and gluinos in events with isolated leptons, jets and missing transverse momentum at $\sqrt{s}=8 \mathrm{TeV}$ with the ATLAS detector, JHEP 04 (2015) 116 [arXiv: 1501.03555] [INSPIRE].

[23] N. Deutschmann, T. Flacke and J.S. Kim, Current LHC Constraints on Minimal Universal Extra Dimensions, Phys. Lett. B 771 (2017) 515 [arXiv:1702.00410] [InSPIRE].

[24] J. Beuria, A. Datta, D. Debnath and K.T. Matchev, LHC Collider Phenomenology of Minimal Universal Extra Dimensions, Comput. Phys. Commun. 226 (2018) 187 [arXiv: 1702.00413] [INSPIRE].

[25] Particle Data Group collaboration, Review of Particle Physics, Phys. Rev. D 98 (2018) 030001 [INSPIRE].

[26] I. Antoniadis, A Possible new dimension at a few TeV, Phys. Lett. B 246 (1990) 377 [INSPIRE].

[27] K.R. Dienes, E. Dudas and T. Gherghetta, Extra space-time dimensions and unification, Phys. Lett. B 436 (1998) 55 [hep-ph/9803466] [INSPIRE].

[28] I. Antoniadis, N. Arkani-Hamed, S. Dimopoulos and G.R. Dvali, New dimensions at a millimeter to a Fermi and superstrings at a TeV, Phys. Lett. B 436 (1998) 257 [hep-ph/9804398] [INSPIRE].

[29] N. Arkani-Hamed, S. Dimopoulos and G.R. Dvali, The Hierarchy problem and new dimensions at a millimeter, Phys. Lett. B 429 (1998) 263 [hep-ph/9803315] [INSPIRE].

[30] L. Randall and R. Sundrum, A Large mass hierarchy from a small extra dimension, Phys. Rev. Lett. 83 (1999) 3370 [hep-ph/9905221] [INSPIRE].

[31] N. Arkani-Hamed, T. Cohen, R.T. D'Agnolo, A. Hook, H.D. Kim and D. Pinner, Solving the Hierarchy Problem at Reheating with a Large Number of Degrees of Freedom, Phys. Rev. Lett. 117 (2016) 251801 [arXiv:1607.06821] [INSPIRE].

[32] K. Agashe, G. Perez and A. Soni, Flavor structure of warped extra dimension models, Phys. Rev. D 71 (2005) 016002 [hep-ph/0408134] [INSPIRE].

[33] S.J. Huber, Flavor violation and warped geometry, Nucl. Phys. B 666 (2003) 269 [hep-ph/0303183] [INSPIRE].

[34] A.L. Fitzpatrick, G. Perez and L. Randall, Flavor anarchy in a Randall-Sundrum model with 5D minimal flavor violation and a low Kaluza-Klein scale, Phys. Rev. Lett. 100 (2008) 171604 [arXiv:0710.1869] [INSPIRE].

[35] T.G. Rizzo, Kinetic mixing, dark photons and an extra dimension. Part I, JHEP 07 (2018) 118 [arXiv: 1801.08525] [INSPIRE].

[36] T.G. Rizzo, Kinetic mixing, dark photons and extra dimensions. Part II: fermionic dark matter, JHEP 10 (2018) 069 [arXiv: 1805.08150] [INSPIRE].

[37] V. Silveira and A. Zee, Scalar phantoms, Phys. Lett. 161B (1985) 136 [InSPIRE].

[38] J. McDonald, Gauge singlet scalars as cold dark matter, Phys. Rev. D 50 (1994) 3637 [hep-ph/0702143] [INSPIRE].

[39] C.P. Burgess, M. Pospelov and T. ter Veldhuis, The Minimal model of nonbaryonic dark matter: A Singlet scalar, Nucl. Phys. B 619 (2001) 709 [hep-ph/0011335] [inSPIRE].

[40] M.C. Bento, O. Bertolami, R. Rosenfeld and L. Teodoro, Selfinteracting dark matter and invisibly decaying Higgs, Phys. Rev. D 62 (2000) 041302 [astro-ph/0003350] [INSPIRE]. 
[41] O. Bertolami and R. Rosenfeld, The Higgs portal and an unified model for dark energy and dark matter, Int. J. Mod. Phys. A 23 (2008) 4817 [arXiv:0708.1784] [INSPIRE].

[42] M.C. Bento, O. Bertolami and R. Rosenfeld, Cosmological constraints on an invisibly decaying Higgs boson, Phys. Lett. B 518 (2001) 276 [hep-ph/0103340] [INSPIRE].

[43] J. March-Russell, S.M. West, D. Cumberbatch and D. Hooper, Heavy Dark Matter Through the Higgs Portal, JHEP 07 (2008) 058 [arXiv: 0801.3440] [INSPIRE].

[44] A. Biswas and D. Majumdar, The Real Gauge Singlet Scalar Extension of Standard Model: A Possible Candidate of Cold Dark Matter, Pramana 80 (2013) 539 [arXiv:1102.3024] [INSPIRE].

[45] R. Costa, A.P. Morais, M.O.P. Sampaio and R. Santos, Two-loop stability of a complex singlet extended Standard Model, Phys. Rev. D 92 (2015) 025024 [arXiv:1411.4048] [INSPIRE].

[46] A. Eichhorn and M.M. Scherer, Planck scale, Higgs mass and scalar dark matter, Phys. Rev. D 90 (2014) 025023 [arXiv: 1404.5962] [inSPIRE].

[47] N. Khan and S. Rakshit, Study of electroweak vacuum metastability with a singlet scalar dark matter, Phys. Rev. D 90 (2014) 113008 [arXiv:1407.6015] [INSPIRE].

[48] F.S. Queiroz and K. Sinha, The Poker Face of the Majoron Dark Matter Model: LUX to keV Line, Phys. Lett. B 735 (2014) 69 [arXiv:1404.1400] [INSPIRE].

[49] C. Kouvaris, I.M. Shoemaker and K. Tuominen, Self-Interacting Dark Matter through the Higgs Portal, Phys. Rev. D 91 (2015) 043519 [arXiv:1411.3730] [InSPIRE].

[50] S. Bhattacharya, S. Jana and S. Nandi, Neutrino Masses and Scalar Singlet Dark Matter, Phys. Rev. D 95 (2017) 055003 [arXiv: 1609.03274] [InSPIRE].

[51] O. Bertolami, C. Cosme and J.G. Rosa, Scalar field dark matter and the Higgs field, Phys. Lett. B 759 (2016) 1 [arXiv:1603.06242] [INSPIRE].

[52] R. Campbell, S. Godfrey, H.E. Logan and A. Poulin, Real singlet scalar dark matter extension of the Georgi-Machacek model, Phys. Rev. D 95 (2017) 016005 [arXiv: 1610.08097] [INSPIRE].

[53] M. Heikinheimo, T. Tenkanen, K. Tuominen and V. Vaskonen, Observational Constraints on Decoupled Hidden Sectors, Phys. Rev. D 94 (2016) 063506 [Erratum ibid. D 96 (2017) 109902] [arXiv: 1604.02401] [INSPIRE].

[54] K. Kainulainen, S. Nurmi, T. Tenkanen, K. Tuominen and V. Vaskonen, Isocurvature Constraints on Portal Couplings, JCAP 06 (2016) 022 [arXiv: 1601.07733] [InSPIRE].

[55] S. Nurmi, T. Tenkanen and K. Tuominen, Inflationary Imprints on Dark Matter, JCAP 11 (2015) 001 [arXiv: 1506. 04048] [inSPIRE].

[56] T. Tenkanen, Feebly Interacting Dark Matter Particle as the Inflaton, JHEP 09 (2016) 049 [arXiv: 1607.01379] [INSPIRE].

[57] J.A. Casas, D.G. Cerdeño, J.M. Moreno and J. Quilis, Reopening the Higgs portal for single scalar dark matter, JHEP 05 (2017) 036 [arXiv:1701.08134] [INSPIRE].

[58] C. Cosme, J.G. Rosa and O. Bertolami, Scalar field dark matter with spontaneous symmetry breaking and the $3.5 \mathrm{keV}$ line, Phys. Lett. B 781 (2018) 639 [arXiv:1709.09674] [InSPIRE].

[59] M. Heikinheimo, T. Tenkanen and K. Tuominen, WIMP miracle of the second kind, Phys. Rev. D 96 (2017) 023001 [arXiv: 1704.05359] [INSPIRE]. 
[60] R.G. Landim, Dark energy, scalar singlet dark matter and the Higgs portal, Mod. Phys. Lett. A 33 (2018) 1850087 [arXiv: 1712.09653] [INSPIRE].

[61] M. Duerr, P. Fileviez Pérez and J. Smirnov, Scalar Dark Matter: Direct vs. Indirect Detection, JHEP 06 (2016) 152 [arXiv:1509.04282] [INSPIRE].

[62] GAMBIT collaboration, Status of the scalar singlet dark matter model, Eur. Phys. J. C 77 (2017) 568 [arXiv: 1705.07931] [INSPIRE].

[63] A. Djouadi, O. Lebedev, Y. Mambrini and J. Quevillon, Implications of LHC searches for Higgs-portal dark matter, Phys. Lett. B 709 (2012) 65 [arXiv:1112.3299] [INSPIRE].

[64] K. Cheung, Y.-L.S. Tsai, P.-Y. Tseng, T.-C. Yuan and A. Zee, Global Study of the Simplest Scalar Phantom Dark Matter Model, JCAP 10 (2012) 042 [arXiv:1207.4930] [INSPIRE].

[65] A. Djouadi, A. Falkowski, Y. Mambrini and J. Quevillon, Direct Detection of Higgs-Portal Dark Matter at the LHC, Eur. Phys. J. C 73 (2013) 2455 [arXiv:1205.3169] [INSPIRE].

[66] J.M. Cline, K. Kainulainen, P. Scott and C. Weniger, Update on scalar singlet dark matter, Phys. Rev. D 88 (2013) 055025 [Erratum ibid. D 92 (2015) 039906] [arXiv:1306.4710] [INSPIRE].

[67] M. Endo and Y. Takaesu, Heavy WIMP through Higgs portal at the LHC, Phys. Lett. B 743 (2015) 228 [arXiv:1407.6882] [INSPIRE].

[68] A. Goudelis, Y. Mambrini and C. Yaguna, Antimatter signals of singlet scalar dark matter, JCAP 12 (2009) 008 [arXiv: 0909.2799] [InSPIRE].

[69] A. Urbano and W. Xue, Constraining the Higgs portal with antiprotons, JHEP 03 (2015) 133 [arXiv: 1412.3798] [INSPIRE].

[70] LUX collaboration, Improved Limits on Scattering of Weakly Interacting Massive Particles from Reanalysis of 2013 LUX Data, Phys. Rev. Lett. 116 (2016) 161301 [arXiv: 1512.03506] [INSPIRE].

[71] X.-G. He and J. Tandean, New LUX and PandaX-II Results Illuminating the Simplest Higgs-Portal Dark Matter Models, JHEP 12 (2016) 074 [arXiv:1609.03551] [INSPIRE].

[72] M. Escudero, A. Berlin, D. Hooper and M.-X. Lin, Toward (Finally!) Ruling Out Z and Higgs Mediated Dark Matter Models, JCAP 12 (2016) 029 [arXiv:1609.09079] [InSPIRE].

[73] Planck collaboration, Planck 2015 results. XIII. Cosmological parameters, Astron. Astrophys. 594 (2016) A13 [arXiv:1502.01589] [INSPIRE].

[74] J.M. Cline and P. Scott, Dark Matter CMB Constraints and Likelihoods for Poor Particle Physicists, JCAP 03 (2013) 044 [Erratum ibid. 05 (2013) E01] [arXiv:1301.5908] [INSPIRE].

[75] T.R. Slatyer, Indirect dark matter signatures in the cosmic dark ages. I. Generalizing the bound on s-wave dark matter annihilation from Planck results, Phys. Rev. D 93 (2016) 023527 [arXiv: 1506.03811] [INSPIRE].

[76] Fermi-LAT collaboration, Searching for Dark Matter Annihilation from Milky Way Dwarf Spheroidal Galaxies with Six Years of Fermi Large Area Telescope Data, Phys. Rev. Lett. 115 (2015) 231301 [arXiv:1503.02641] [INSPIRE].

[77] LUX collaboration, Results from a search for dark matter in the complete LUX exposure, Phys. Rev. Lett. 118 (2017) 021303 [arXiv: 1608.07648] [INSPIRE]. 
[78] PandaX-II collaboration, Dark Matter Results from First 98.7 Days of Data from the PandaX-II Experiment, Phys. Rev. Lett. 117 (2016) 121303 [arXiv:1607.07400] [InSPIRE].

[79] SuperCDMS collaboration, Search for Low-Mass Weakly Interacting Massive Particles with SuperCDMS, Phys. Rev. Lett. 112 (2014) 241302 [arXiv:1402.7137] [INSPIRE].

[80] XENON100 collaboration, Dark Matter Results from 225 Live Days of XENON100 Data, Phys. Rev. Lett. 109 (2012) 181301 [arXiv:1207.5988] [inSPIRE].

[81] ICECuBE collaboration, Search for dark matter annihilations in the Sun with the 79-string IceCube detector, Phys. Rev. Lett. 110 (2013) 131302 [arXiv:1212.4097] [INSPIRE].

[82] ICECUBE collaboration, Improved limits on dark matter annihilation in the Sun with the 79-string IceCube detector and implications for supersymmetry, JCAP 04 (2016) 022 [arXiv: 1601.00653] [INSPIRE].

[83] G.R. Dvali and S.H.H. Tye, Brane inflation, Phys. Lett. B 450 (1999) 72 [hep-ph/9812483] [INSPIRE].

[84] A. De Rujula, A. Donini, M.B. Gavela and S. Rigolin, Fat brane phenomena, Phys. Lett. B 482 (2000) 195 [hep-ph/0001335] [INSPIRE].

[85] H. Georgi, A.K. Grant and G. Hailu, Chiral fermions, orbifolds, scalars and fat branes, Phys. Rev. D 63 (2001) 064027 [hep-ph/0007350] [inSPIRE].

[86] R. Foot, New Physics From Electric Charge Quantization?, Mod. Phys. Lett. A 6 (1991) 527 [INSPIRE].

[87] X.G. He, G.C. Joshi, H. Lew and R.R. Volkas, New Z-prime phenomenology, Phys. Rev. D 43 (1991) 22 [INSPIRE].

[88] X.-G. He, G.C. Joshi, H. Lew and R.R. Volkas, Simplest Z-prime model, Phys. Rev. D 44 (1991) 2118 [INSPIRE].

[89] M. Bauer, P. Foldenauer and J. Jaeckel, Hunting All the Hidden Photons, JHEP 07 (2018) 094 [arXiv: 1803.05466] [INSPIRE].

[90] K. Petraki and R.R. Volkas, Review of asymmetric dark matter, Int. J. Mod. Phys. A 28 (2013) 1330028 [arXiv: 1305.4939] [INSPIRE].

[91] G.R. Dvali, G. Gabadadze and M.A. Shifman, (Quasi)localized gauge field on a brane: Dissipating cosmic radiation to extra dimensions?, Phys. Lett. B 497 (2001) 271 [hep-th/0010071] [INSPIRE].

[92] M. Carena, T.M.P. Tait and C.E.M. Wagner, Branes and Orbifolds are Opaque, Acta Phys. Polon. B 33 (2002) 2355 [hep-ph/0207056] [INSPIRE].

[93] Planck collaboration, Planck 2018 results. VI. Cosmological parameters, arXiv: 1807.06209 [INSPIRE].

[94] R. Essig, J. Mardon and T. Volansky, Direct Detection of Sub-GeV Dark Matter, Phys. Rev. D 85 (2012) 076007 [arXiv:1108.5383] [INSPIRE].

[95] A. Berlin, D. Hooper and S.D. McDermott, Simplified Dark Matter Models for the Galactic Center Gamma-Ray Excess, Phys. Rev. D 89 (2014) 115022 [arXiv:1404.0022] [INSPIRE].

[96] T. Emken, C. Kouvaris and I.M. Shoemaker, Terrestrial Effects on Dark Matter-Electron Scattering Experiments, Phys. Rev. D 96 (2017) 015018 [arXiv:1702.07750] [INSPIRE]. 
[97] XENON10 collaboration, A search for light dark matter in XENON10 data, Phys. Rev. Lett. 107 (2011) 051301 [Erratum ibid. 110 (2013) 249901] [arXiv : 1104. 3088] [INSPIRE].

[98] XENON collaboration, Low-mass dark matter search using ionization signals in XENON100, Phys. Rev. D 94 (2016) 092001 [Erratum ibid. D 95 (2017) 059901] [arXiv: 1605. 06262] [INSPIRE].

[99] DARKSide collaboration, Constraints on Sub-GeV Dark-Matter-Electron Scattering from the DarkSide-50 Experiment, Phys. Rev. Lett. 121 (2018) 111303 [arXiv:1802.06998] [INSPIRE].

[100] SENSEI collaboration, SENSEI: First Direct-Detection Constraints on sub-GeV Dark Matter from a Surface Run, Phys. Rev. Lett. 121 (2018) 061803 [arXiv:1804.00088] [INSPIRE].

[101] ATLAS collaboration, Search for invisible Higgs boson decays in vector boson fusion at $\sqrt{s}=13 \mathrm{TeV}$ with the ATLAS detector, Phys. Lett. B 793 (2019) 499 [arXiv:1809.06682] [INSPIRE].

[102] ATLAS collaboration, Constraints on new phenomena via Higgs boson couplings and invisible decays with the ATLAS detector, JHEP 11 (2015) 206 [arXiv:1509.00672] [INSPIRE]. 